\title{
Der Stellenwert von Subjektivität in der Medizin - Diagnose und Therapie unter Einbezug empathischer Wahrnehmung durch Arzt und Therapeut
}

\author{
Markus Köhl \\ Medizinische Fakultät der Universität Witten-Herdecke, Zentrum für Elektropathologie und Umweltmedizin, Witten, Deutschland
}

$\mathrm{D}$ er Mediziner und Sinnesphysiologe Herbert HENSEL trat in den 80er Jahren gemeinsam mit dem Mediziner VIKTOR v. WEIZSÄCKER für den bedeutenden Stellenwert der Subjektivität in der Medizin und Forschung ein [1]. Die Korrelation von subjektiven Sinnesempfindungen mit objektiven Messwerten in ihrer wissenschaftlichen Arbeit auf dem Gebiet der Psychophysik führte zu der Erkenntnis, dass das Subjektive als unmittelbare Erfahrung zum Handwerkszeug der medizinischen Diagnose und Behandlung gehört. Dies wird in einem medizinischen System, das sich technisch durch immer aufwändigere diagnostische Verfahren auszeichnet und sich vornehmlich einer objektiven Seite zuwendet, nur möglich, wenn die bewusste Überzeugung für die Einbeziehung des subjektiven Blicks und Empfindens vorliegt [2]. Es gehören jedoch Vertrauen und Stabilität des Arztes dazu, seinen eigenen subjektiven Körperempfindungen und Sinneseindrücken zu vertrauen und diese mit einzubeziehen. In einer Forschungsarbeit werden vegetative und zerebrale Prozesse für die Gestaltung der subjektiven Wahrnehmungsfähigkeiten (u.a. visuell, auditiv, sensorisch-palpatorisch und empathisch-atmosphärisch) betrachtet, die den Zugang zur bewussten Wahrnehmung ermöglichen [3].

\section{Praxisalltag des Arztes und Therapeuten}

Naturwissenschaftlich-technisch orientiertes Denken verlässt sich im Zweifelsfall nicht auf Gefühle oder den Instinkt, sondern vertraut eher technischen Befunden als der persönlichen
Hintergrund: Die Veränderung der Hirnaktivität und der vegetativen Funktionen während eines erweiterten Wahrnehmungseindrucks sind Thema einer Forschungsarbeit an der Universität WittenHerdecke. Die untersuchte Wahrnehmungsleistung wird auf die Qualität einer Arzt-Patientenbeziehung übertragen. In dieser Beziehung spielt die Empathie eine ausschlaggebende Rolle. Dabei steht die empathische Wahrnehmung für eine gesamtheitliche Sicht auf den Patienten, wobei seine Bedürfnisse und Ressourcen samt seiner Situation umfassender erkannt werden können. Erhöhte Wahrnehmungsqualität zeigt im übertragenen Sinn, dass der Arzt an Kompetenz gewinnt, wenn er eigene mentale Ressourcen empfindet und subjektive Eindrücke in die Diagnose mit einbezieht. Studienziel: Ziel der Studie ist es, den Einfluss des vegetativen und zerebralen Zustandes auf die Qualität und das Spektrum von Wahrnehmungseindrücken hin zu analysieren. Die Ergebnisse der körperlichen und psychologischen Verfassung werden auf das empathiefähige Handeln bezogen. Methodik: Es wurde eine Entspannungsmethode in Kombination mit einem Wahrnehmungsversuch angewendet und die vegetativen und zerebralen Messverläufe wurden mit der Bewertung der Wahrnehmungsqualität korreliert. Dazu wurde ein Vorversuch ohne Entspannung und eine Hauptstudie mit Entspannung durchgeführt, jeweils mit 33 Probanden mittleren Alters (25,9 Jahre). Durch eine Mittelwertberechnung wurde der Verlauf der vegetativen und zerebralen Parameter festgestellt und daraus ein Vegetativportrait erstellt. Der Verlauf der Mittelwerte wurde mit der jeweiligen Wahrnehmungsleistung der Probanden korreliert und die Fähigkeit des sensomotorischen Empfindens und des visuellen Erkennens durch eine hohe (WQ1-2) oder niedrige (WO3-4) Wahrnehmungsqualität kategorisiert. Mit der Berechnung der Differenz der vegetativen und zerebralen Mittelwerte in den vier Versuchsphasen wird die Flexibilität, Stabilität oder Starre und Unausgewogenheit des jeweiligen Systems während veränderter Wahrnehmung untersucht. Ergebnisse: Es ist gelungen, die Wahrnehmungsqualität und deren Sensibilität mit den vegetativen und zerebralen Messwerten im Vegetativportrait zu korrelieren. Während empathiefähigem Wahrnehmen zeigt sich ein charakteristisches Vegetativum. Dabei konnte ein Trend für die Charakteristik im Vegetativum während wacher und entspannter Aufmerksamkeit festgestellt und ein relevanter Unterschied zwischen den Probanden mit hoher und niedriger Wahrnehmungsleistung aufgezeigt werden. Die Korrelation von vegetativen, zerebralen Funktionszuständen während einer erweiterten Wahrnehmung ergibt tendenziell typische Verläufe im Vegetativum, im EEG ein spezielles Frequenzspektrum und einen signifikanten kortiko-kortikalen, globalen Kohärenzanstieg. Dabei wurde die Aufhebung der Alpha-Blockade festgestellt. Schlussfolgerung: Eine ruhige, innere Gelassenheit und mentale Vorbereitung des Arztes und Therapeuten fördert die Empathiefähigkeit, auf der eine Gestaltung der Arzt-/Therapeuten-Patienten-Beziehung aufbaut. Erweiterte Wahrnehmungsqualität und Erhöhung der Empathiefähigkeit werden durch vegetative Ausgewogenheit und zerebrale Flexibilität gefördert. Dies schafft den bewussten Zugang zu weitem, subjektivem Wahrnehmen des Arztes oder Therapeuten von sich selbst und dem Patienten und unterstützt ein vertrauensvolles Klima in der Arzt-Patienten-Beziehung.

Schlüsselwörter: Empathie, Arzt-/Therapeuten-Patienten-Beziehung, Wahrnehmung, vegetative und zerebrale Entspannung, kortiko-kortikale globale Kohärenz, vertieftes Bewusstsein, kreativer Werkprozess

The Significance of Subjectivity in Medical Practice - Empathic Perception for Diagnosis and Therapy by the Physician/Therapist

Background: Change in brain activity and vegetative functions during expanded perception have been the focus of a research project at Witten-Herdecke University. Perceptual performance is then applied to the quality of a doctor-patient relationship. Empathy plays a decisive role in this relationship, and empathic perception represents a holistic view of the patient enabling a more comprehensive identification of his or her needs, resources and situation. In an applied sense, a higher quality of perception demonstrates that the physician gains competence if he or she is sensible of his own mental resources and integrates subjective impressions into the diagnosis. Aim of the study: The aim of the study was to analyse the influence of the vegetative and cerebral state on the quality and spectrum of perceptual impressions. Conclusions drawn on the physical and psychological constitution are related to the capacity for empathy. Methods: A relaxation method was combined with a perceptual experiment and the recorded vegetative and cerebral 
measurements were correlated with the evaluation of quality of perception. To this end, a pretrial without relaxation and a main study with relaxation were carried out, each with 33 test participants of a median age of 25.9 years. The measurement curve of the vegetative and cerebral parameters was determined by calculating averages; from this a vegetative portrait was generated. The curve of the average values was correlated with the perceptual performance of the subjects, and their capacity for sensorimotor perception and visual recognition was categorised according to a high (RQ1-2) or a low (RO3-4) perceptual quality. In order to investigate the flexibility and stability or rigidity and imbalance of the respective systems during changed perception, the difference between the vegetative and cerebral average values in the four test phases was calculated. Results: The quality of perception and perceptual sensibility were successfully correlated to the vegetative and cerebral measurements in the vegetative portrait. A characteristic autonomic nervous system was displayed during empathetic perception. At the same time, a trend emerged concerning the characteristics in the autonomic nervous system during alert and relaxed attention, and a relevant difference was registered between subjects with higher and lower perceptual performance. The correlation between vegetative, cerebral functional states during expanded perception produces a tendency towards typical measurement curves in the autonomic nervous system, a particular frequency spectrum in the EEG, and a significant increase in global corticocortical coherence. Alpha blockade disappeared during this process. Conclusions: The calm, inner composure and mental preparation of the physician and therapist foster his empathic capacity, which serves as the basis for developing the physician/therapist-patient relationship. Expanded quality of perception and an increased capacity for empathy are promoted by vegetative balance and cerebral flexibility. In the physician or therapist, this creates conscious access to broad, subjective perception of self and of the patient, and fosters an atmosphere of trust in the physicianpatient relationship.

Key words: Empathy, physician/therapist-patient relationship, perception, vegetative and cerebral relaxation, global cortico-cortical coherence, heightened awareness, creative work process

Wahrnehmung [4]. Seelische Aspekte der Gesundheitsfürsorge gehören neben modernster Diagnose- und Behandlungsverfahren als intraprofessioneller Teil zu einer ganzheitlichen Medizin, die nicht nur medizinische Daten, sondern auch die Persönlichkeit und die individuelle Situation des Patienten erfasst [5]. Eine patientenorientierte Gesprächsmedizin, die den Menschen und seinen Körper als eine Einheit betrachtet und der Kommunikation in der Behandlung mehr Raum gibt, kann dies möglicherweise verwirklichen helfen [6].

\section{Wahrnehmungserweiterung für intensives empathisches Handeln}

Ein kreativer Geist dient dem Arzt dazu, Fachwissen, Intuition und Beobachtung für eine therapeutische Empfehlung zu verbinden. Während der Befundung eines Patienten kann durch eine erweiterte Wahrnehmung neben standardisiertem Fachwissen auch die intuitive, kreative Wahrnehmungsleistung eingebracht werden, um eine umfassende Diagnose zu erstellen. Der Arzt steht immer wieder vor Neuem, zunächst Unbekanntem. Zu individuell und verschieden ist die Krankheitsgeschichte eines jeden Patienten, als dass ausschliesslich allgemeingültige, statistische Definitionen und Normwerte angewandt werden können [7].
Arzt und Therapeut erfahren durch das Hinzuziehen der phänomenologischen Betrachtung den eigenen $\mathrm{Zu}$ stand und ihre körperliche und geistige Verfassung und können durch Selbstreflexion und innere Neutralität und Offenheit eine höhere empathische Qualität entwickeln. Bei der ganzheitlich orientierten Sichtweise der Salutogenese richtet sich der Wahrnehmungsfokus auf sämtliche Lebens- und Seinsbereiche des Menschen und betrachtet ihn als Gestalter seines Lebens mit all seinen gesunden wie kranken Anteilen, anstatt lediglich auf die Beseitigung der Symptome zu achten.

Zwischen Gesundheit und Krankheit liegt ein fliessender Übergang, der mit der rein wissenschaftlichen Sichtweise nicht erkannt werden kann [8]. Gesundheit kann nur geahnt werden, um sie im nächsten Schritt fördern zu können. Zur Erfassung des Patienten und seines Zustandes ist daher vertiefte Wahrnehmung nötig, die das dynamische Zusammenspiel von Gegensätzen betrachtet und das Potential für einen Gesundheitszustand erkennt. Um den Zugang zur Gesundheit zu finden, bedarf es einer guten Eigenwahrnehmung bzw. Körperwahrnehmung. Um diese zu entwickeln, ist es hilfreich die Aufmerksamkeit auf den Atem (Rhythmus, Atemtiefe und Atemräume) $\mathrm{zu}$ richten, sowie auf die Kör- perhaltung zu achten und deren Spannungsbalance/Disbalance zu erspüren. Der Behandler kann darüber hinaus seinen Berührungs- und Tastsinn verfeinern, um die Qualität der Gewebespannung, Beweglichkeit des Gewebes und den Rhythmus der Flüssigkeiten im Gewebe wahrzunehmen. Dadurch kann ein Befund erhoben werden.

Arzt und Therapeut achten dabei mit einer phänomenologisch-empathischen Wahrnehmung auf die körperliche und geistige Verfassung des Patienten, auf seine Körperhaltung, Mimik, Sprache und körperlichen Signale, wie Anspannung und Entspannung, Atmung oder Herzschlag. Das Erfassen dieser komplexen Wahrnehmungsinhalte gibt dem Arzt und Therapeuten Orientierung und zusätzliche Kompetenz für sein Handeln [9].

Während einer Erweiterung der Wahrnehmung intensiviert sich die Empfindungsfähigkeit, die es vermag, umfassend die Gesamtzusammenhänge $\mathrm{zu}$ erkennen und verborgene, kreative Kräfte beim Patienten zu aktivieren und damit gesunde Persönlichkeitsanteile zu stärken. In der aktuellen Forschung von BRIESKORN-ZINKE, die sich auf das Antonovsky-Modell der Salutogenese [10] bezieht, besteht ein Beitrag des Arztes und Therapeuten darin, das Gesundheits-Krankheits-Kontinuum des Patienten zu lokalisieren und ihn dann beim Vorwärtskommen in Richtung Gesundheitspol zu unterstützen [11]. Salutogenetisch orientierte Medizin stellt kein alternatives, separates Modell dar, sondern ist eine ergänzende Betrachtungsweise in der Medizin [12].

\section{Empathisches Handeln} beinhaltet sensibles Wahrnehmen

Empathie steht für unterschiedliche Fähigkeiten und Zustände des Therapeuten in Bezug auf die Wahrnehmung seines Gegenübers [13]. Sie umschreibt Kommunikationsfähigkeit, wie Zuhören, ausgewogen und vermittelnd Kommunizieren, bewusste Körpersprache zur Beziehungsaufnahme und Vertrauensbildung [14]. Sie beinhaltet auch Einfindungsvermögen, Neutralität und Integrität anstelle von Dominanzen. Bei der Empathie geht es um Einfühlung in Menschen. Ein oberflächlicher Blick reicht nicht aus, will man $\mathrm{Pa}$ - 
tienten wirksam helfen. Empathie beinhaltet, in jedem Augenblick ein Gespür zu haben für die sich ändernden, fixierenden und subjektiven Bedeutungen in dieser Person, um ihr dann auch wirklich ganz individuell helfen zu können. Dies bedeutet für die heutige Patienten- und Arbeitsfrequenz des Praxis- und Klinikalltags eine hilfreiche Ergänzung und kann den Arzt und Therapeuten in ihrem Arbeitsalltag dabei unterstützen, mehr Wohlbefinden $\mathrm{zu}$ erhalten.

\section{Selbstwahrnehmung des Arztes durch erweiterte Wahrnehmung}

Eine geschulte Selbstwahrnehmung ist der Schlüsselfaktor zur erweiterten Wahrnehmung und empathischer Fähigkeit [15]. Dies sind gleichzeitig gesundheitserhaltende Massnahmen und Methoden zur Stärkung des Lebensgefühls. Das Gefühl der Bedeutsamkeit und Sinnhaftigkeit der Arbeit kann gepflegt werden, wenn Raum zur Reflexion genommen wird. Dies stärkt Phasen emotional belastender Momente und kann somit einem Burn-out vorbeugen [16]. Um psycho-vegetativer Erschöpfung vorzubeugen, braucht es eine Selbstpflegekompetenz. Diese notwenige Haltung hängt mit innerer Versammlung und Achtsamkeit zusammen, um überhaupt das Empathievermögen für sich und seine Patienten zu steigern und auszubauen.

$\mathrm{Zu}$ einem ausgeprägten Empathievermögen gehören Offenheit, psychische Stabilität und gesunde Selbstsicherheit, Reflexionsfähigkeit sowie mentale Gelassenheit. Als weitere Säule der erweiterten Wahrnehmung werden das Selbstmanagement und die Selbstmotivation definiert, das Aussen mit sich selbst in Gleichklang zu bringen, die Balance zwischen Kopf und Bauch.

\section{Erweiterte Wahrnehmungsqualität für einen salutogenesischen Blick in Diagnose und Behandlung}

Eine wachsende Empathiefähigkeit ist Kennzeichen einer erweiterten Wahrnehmung. In einem entspannten Wachzustand werden subtilere Eindrücke wahrgenommen, die sonst unbemerkt bleiben oder nur unterschwellig, nicht auf den ersten Blick erkannt werden. Bei einer vertieften empathischen Be- ziehung wird das Bewusstsein für das Wecken von eigenen Ressourcen des Patienten verstärkt und seine Fähigkeiten, sein Können, seine gesunden Anteile, d.h. sein „gesunder Kern“ erfahrbar gemacht.

Es geht bei der Betrachtung von Gesundheit um die kreativen, in Bewegung befindlichen Prozesse im Inneren und Äusseren des Menschen. Gesundheit kommt aus dem Unbekannten, daher ist sie nicht ausschliesslich zu objektivieren und messbar oder beschreibbar einzugrenzen. Gesundheit ist nach HANS-GEORG GADAMER [17] wesensmässig verborgen. Der Mediziner KLAUS DöRNER nennt sie ein Sich-Entziehendes, ein Abwesendes, ein Weggegebenes [18]. Eine intensivierte Wahrnehmungsqualität betrachtet das $\mathrm{Zu}$ sammenspiel und Spannungsfeld von Gesundheit und Krankheit und erspürt das dynamische Gleichgewicht der körperlichen und geistigen Polaritäten. Die innere Haltung des Ahnens schafft eine Öffnung und Neutralität, so dass sich das Unbekannte der subjektiven Wahrnehmung unmittelbar erschliesst. Rein willentlich, voreingenommen mit festen Wertungen und Vorstellungen bleibt die phänomenologische Sichtweise verschlossen. Messergebnisse zeigen auf, wie das Bewertungssystem bei einem empathiefähigen Verhalten in den Hintergrund rückt und die Offenheit im Wahrnehmen zunimmt.

Unterschiedliche Phänomene können beispielsweise in Form von körperlichem und geistigem Wohlempfinden, Wärme, freier Nase und Durchatmen-Können, klaren Gedanken und unbeschwerten Gefühlen, Anspannung und Entspannung, Leichtigkeit und Schwere, Entlastung und Nachlassen von innerem Druck wahrgenommen werden. Der Begriff Phänomen, der hier verwendet wird, ist der der naturalistischen, subjektiven Erscheinung, der allerdings eine tiefere, aber nicht unbedingt eine logisch-rational erfassbare Wahrheit zugrunde liegen kann. Der phänomenologische Ansatz bezieht sich auf den Erfahrungsbereich des subjektiven Erlebens.

Kreativität in der Wahrnehmung als gesundheitsfördernde Betrachtungsweise zeigt sich bei analytischem und ahnend schauendem Handeln, bei dem eine Symbiose von Wissen und Unbekanntem stattfindet [19]. Das zuvor ausschliesslich fachspezifisch-medizinische Anliegen bekommt eine zusätzliche Akzentuierung und Ausweitung durch einen freien Geisteszustand, der zuvor rational gegründet ist, aber sich öffnen und von festen Vorstellungen loslassen kann. So wird das Unbekannte trotz des Wissens erfahrbar [20].

\section{Methodik}

Das Forschungsprojekt untersucht das empathiefähige Empfindungs- und Einfühlungsvermögen unter bestimmten vegetativen und zerebralen Voraussetzungen unter dem Gesichtspunkt einer Wahrnehmungsqualität, die sich auf phänomenologische Wahrnehmungseindrücke bezieht. Die Versuchsanordnung beinhaltet eine körperliche Entspannung und anschliessend eine kognitive Aufgabe zur Messung der Wahrnehmungsleistung. Dabei wird ein Vegetativportrait erstellt und die Veränderung der Hirnaktivitäten gemessen. Die Ergebnisse werden mit der Wahrnehmungsqualität korreliert, um die zerebralen Funktionsmechanismen verstehen zu können.

\section{Hypothese}

Es soll überprüft werden, ob einer erweiterten Wahrnehmung eine wache und entspannte Aufmerksamkeit zu Grunde liegt. Dabei wurde untersucht, ob die Wahrnehmungsqualität in $\mathrm{Zu}$ sammenhang mit physiologischen, vegetativen und zerebralen Parametern steht und ob man durch Entspannung in der Lage ist, die Qualität der Wahrnehmung zu beeinflussen.

\section{Studiendesign}

Es wurden zwei Versuchsreihen durchgeführt, die einen Vergleichswert zwischen Wahrnehmungsqualität mit und ohne Entspannung ergeben. In einem Vorversuch musste eine Gruppe ohne Entspannung eine kognitive Aufgabe lösen. Danach wurde in einem Hauptversuch die gleiche Probandenzahl wie im Vorversuch auf deren Wahrnehmungsqualität hin untersucht. Für beide Versuchsreihen wurden jeweils 33 Probanden im Alter von 20-30 Jahren, dar- 
unter 11 Männer und 22 Frauen für die Auswertung ausgewählt. Das Durchschnittsalter betrug 25,9 Jahre. Die Probanden setzten sich aus Studenten der medizinischen Fakultät der Universität Witten-Herdecke zusammen.

Die Versuchsanordnung bestand aus einer Entspannungsphase, einem Wahrnehmungsversuch sowie vor und nach der Entspannung aus einer Ruhephase. Zur Untersuchung der Veränderung der Wahrnehmungsqualität während eines entspannten Zustandes wurde exemplarisch die komplementär-therapeutische Massnahme Ergosoma als Entspannungsmethode angewendet. Dies ist eine Entspannungsmethode, die ihre Wurzeln in den traditionellen Heilmethoden des alten Asiens hat. (siehe Kasten mit Erklärung zur Methode). Die Probanden sollten mit der Anwendung in einen möglichst einheitlichen Ruhezustand geführt werden, so dass vergleichbare Voraussetzungen für den anschliessenden Wahrnehmungsversuch gegeben waren. Der Entspannungsprozess wurde anhand der Messungen des Vegetativ-Portraits durch die Parameter wie Herzaktivität (EKG), Hautwiderstand (EDA), Atmung, SympathikusVagus-Aktivität (SVB) und Hirnstrommessung (EEG) festgestellt.
Zur Bewertung der Wahrnehmungsleistung unter dem Einfluss der Entspannung wurde die Doppelbildmethode mit den Tafeln von Chartres (Abb. 1) verwendet [21]. Die Tafeln von Chartres sind ein Doppelbild, wie es häufig in der Neurowissenschaft verwendet wird, um einen binokularen Streit auszulösen [22]. Bei den Tafeln von Chartres können zwei gleichförmige, verschiedenfarbige, rote und blaue, waagrecht angeordnete Kreise visuell zuerst nicht zu einem Bildeindruck zusammengebracht werden, sondern befinden sich in einem ständigen Wechsel. Die Phänomene des Doppelbildes der Tafeln von Chartres sind nur mit einer hohen Wahrnehmungsleistung zu erkennen, so die Annahme. Bei den Tafeln von Chartres gelingt es, bei einer hohen Wahrnehmungsleistung beide Bilder zu einem einheitlichen Gesamtbildeindruck fusionieren zu lassen. Bei hoher Wahrnehmungsleistung können beide Kreise zu einem neuen, phänomenalen Farbeindruck fusioniert werden. Dies geschieht bei einem entspannten Weitblick, der nicht fokussiert und eine Gesamtansicht mit allen Details wahrnimmt, also mit einer inneren offenheit des Betrachters. Je nach Grad der einsetzenden Kreativität verändert sich der Eindruck des Bildes fortwäh-

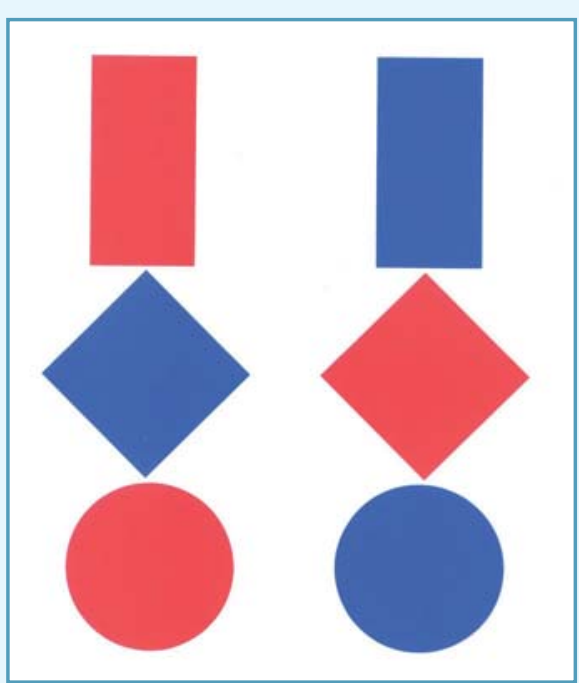

Abb. 1. Tafeln von Chartres. Hier in einer vereinfachten Abbildung, denn die Tafeln von Chartres wurden für den Wahrnehmungsversuch auf das Kreismotiv reduziert, da die Originalabbildung zu komplex für einen Wahrnehmungsversuch ist, bei dem die Probanden keine Vorübung in der Betrachtung eines Doppelbildes haben.

rend weiter. Bei einer hohen Wahrnehmungsqualität werden drei Kreise mit einer neuen Mischfarbe gesehen, die im Verlaufe der Betrachtung dreidimensional erscheinen können. Eine niedrige und höhere Wahrnehmungsqualität (WQ) wurde daraufhin in vier Gruppen kategorisiert, je nachdem welche visuellen Phänomene sich bei der Betrachtung ergaben. Bei sehr guter Wahrnehmungsqualität (WQ 1-2) der Seher werden sehr schnell nach wenigen Sekunden drei Kreise mit einem violetten mittleren Kreis gesehen und bei den Nichtsehern mit niedriger Wahrnehmungsqualität (WQ 3-4) werden nur zwei Kreise und verspätet höchstens vier Kreise erkannt. Grundvoraussetzung für das Gelingen des Wahrnehmungsversuchs mit den Tafeln von Chartres ist die offene, vorurteilsfreie und unvoreingenomme, vigilante Aufmerksamkeit für äussere Reize, die frei von Bewertungskategorien die Umwelt wahrnimmt. Offenheit und Entspannungsvermögen befähigen zur Empathie.

\section{Messinstrumente}

Für die Auswertung und Erstellung des Vegetativportraits wurde das multiparametrische Messverfahren Somno- 
Screen eingesetzt, das in der Schlafforschung etabliert ist. Durch dieses Messverfahren können die vegetativen Veränderungen wie Herz- und Atemfrequenz, Hautwiderstand, Blutdruck, Sympathiko-Vagale-Balance und Herzfrequenzvariabilität angezeigt und im EEG (10-10-System) der Entspannungszustand und die Veränderungen der Hirnaktivität während der Wahrnehmungsphase aufgezeichnet werden. Mit dem EEG-Gerät Neurofax von Nihon Kohden (10-20-System) wurde zusätzlich ein Brain-Mapping durchgeführt, um die Hirnaktivität während der vertieften Wahrnehmung genauer zu lokalisieren. Um die Wahrnehmungsqualitäten im Moment des Ereignisses zu untersuchen, wurde die subjektive Erfahrung durch einen Marker (Handdrücker) und einen mündlichen Erfahrungsbericht der Probanden dokumentiert.

Aus der Messmethode mit den Tafeln von Chartres ergaben sich drei Wahrnehmungsgruppen, mit einer sehr guten, mittleren und schlechten Wahrnehmungsqualität (WQ1, WQ2, WQ 3-4). Die Tafeln dienen der Bewertung und einer Gruppenbildung der Probanden nach Wahrnehmungsleistung. Die Messergebnisse zur Wahrnehmungsqualität sind als psychischer, subjektiver Teil der Arbeit zu bewerten. Je nach Grad der Wahrnehmungsqualität verändert sich der Eindruck des Bildes fortwährend, der durch den Marker in den Messreihen zeitlich vermerkt wird.

\section{Datenanalyse}

Zwei methodische Verfahren beleuchten Entspannung und Wahrnehmung. Zum einen auf der messbar objektiven, physiologischen Ebene, sowie auf der subjektiven Ebene. Auf physiologischer Ebene werden Herz-Kreislaufwerte und zerebrale Werte für den vegetativen, mentalen Zustand gemessen. Zudem wird auf der subjektiven Ebene die Wahrnehmungsqualität als psychischer Teil der Arbeit anhand eines Markers und die mündliche Beschreibung aus den Erfahrungsprotokollen erfasst. Die Wahrnehmungsqualität wird mit den vegetativen und zerebralen Messergebnissen in vier Messphasen vor, während und nach der Entspannungsphase und während des Wahrnehmungsversuches korreliert.
Als Gesamtauswertung der Veränderung und des Verlaufs der vegetativen und zerebralen Parameter findet ein Vergleich der vier Messphasen durch eine Mittelwertbestimmung statt.

Neben dem Verlauf der Mittelwerte wurde die Abweichung bzw. Differenz des Mittelwertes analysiert, um die Veränderung des Vegetativums und des Zerebralen zu bewerten. Es kann festgestellt werden, ob ein System ausgewogen, somnolent oder hyperaktiv funktioniert und die geistigen Funktionen starr oder flexibel auf die Anforderungen reagieren.

Die Deskription der in den vier Versuchsphasen erfassten Merkmale erfolgte tabellarisch durch Angabe von Mittelwert (Mw), Standardabweichung (Sd), Median sowie Minimum und Maximum (Min-Max), bei Darstellung der Verteilung der Daten in Abhängigkeit von der Versuchsphase und der Wahrnehmungsqualität.

Die weitere statistische Auswertung der vegetativen Roh- und Analysedaten wie Herzfrequenz, elektrischer Hautwiderstand und der Messergebnisse aus dem EEG geschah nach folgendem Schema: Die Mittelwerte der ersten Messphasen von Männern und Frauen wurden mit dem t-Test verglichen. Der F-Test wurde in einer einfaktoriellen Varianzanalyse verwendet, um den Einfluss der Wahrnehmungsqualität auf die Untersuchungsergebnisse der ersten Messphase zu untersuchen. Um zu beurteilen, ob sich die Messergebnisse im Versuchsverlauf verändern und inwieweit die Wahrnehmungsqualität die Merkmale und deren Veränderung während des Versuches beeinflusst, wurde eine Varianzanalyse mit zufälligen Effekten und Messwiederholungen berechnet. Hierbei wurde als statistischer Test ebenfalls der F-Test verwendet.

Alle statistischen Tests erfolgten zweiseitig zum Signifikanzniveau 0,05. Wenn der p-Wert kleiner als das Signifikanzniveau ist, dann unterscheiden sich die Gruppen signifikant bzw. es besteht ein signifikanter Effekt. Eine Vigilanzanalyse überprüfte die Signifikanz der Messergebnisse in Bezug zur Wahrnehmungsqualität. Für die Datenaufbereitung und die statistische Analyse wurde Stata/IC Windows benutzt.

\section{Ergebnisse}

Die wissenschaftliche Untersuchung zeigt, dass ausgewogene, stabile vegetative Entspannung und ein spezielles Wellenspektrum im EEG während eines offenen Aufmerksamkeitszustandes [23] sowie eine kortiko-kortikale globale Kohärenz im EEG, also die Vernetzungseigenschaft in der Gehirnrinde, auf die Wahrnehmungsfähigkeit der Probanden förderlich wirkt [24]. Die Kohärenz wird hier im Sinne gleichzeitiger Funktionszustände an unterschiedlichen Orten des Gehirns, in diesem Falle die gleich stark ausgeprägte Gehirnaktivität der linken und rechten Gehirnhälften, genutzt. Kohärenz der Hirnaktivität kann unterschiedlich interpretiert werden. Es gibt die globale Kohärenz, die als ein Hemisphärengleichgewicht über den gesamten Kortex als Untersuchungsgegenstand verstanden wird. Es gibt zudem die lokale Kohärenz, die sich auf spezielle Hirnbereiche und die Vernetzungsaffinität in dem Areal bezieht. Der Hirnforscher H. Petsche nennt es die funktionelle Kopplung von Hirnarealen [25]. Bei hoher kortiko-kortikaler Kohärenz sind die Aktivitäten der linken und rechten Hemisphäre entweder auf einer oder mehreren der Ebenen frontal, zentral, parietal oder sie sind okzipital ausgeglichen oder auf allen vier Ebenen zugleich global ausbalanciert [26].

In einem Vorversuch wurde die Wahrnehmungsqualität ohne Entspannung gemessen. Dabei ergab sich, dass 7\% der Probanden das Wahrnehmungsphänomen der drei Kreise mit einem violetten Mittelkreis erkennen konnten und damit eine gute Wahrnehmungsqualität besassen. Bei der Versuchsreihe mit Entspannung durch die Ergosoma-Methode betrug die Zahl der Probanden mit einer verfeinerten Wahrnehmungsqualität über $81 \%$. Ein Entspannungseinfluss ist somit vor Beginn des Wahrnehmungsversuches erkennbar, der eine Voraussetzung für die erweiterte Wahrnehmung darstellt, wie der Vergleich zum Blindversuch belegt.

\section{Entspannungszustand des Vegetativums}

Es zeigte sich während der Entspannungsphase mit Ergosoma auf körper- 
licher Ebene eine niedrigere Herzrate (Elektrokardiogramm EKG), Zunahme der Herzfrequenzvariabilität (HRV: Eigenschaften der Herzperioden), ansteigende Sauerstoffsättigung, Sauerstoffpuls $\left(\mathrm{O}_{2}\right.$-Gehalt), verbesserte kapillare Durchblutung, gemessen an dem Fingerpuls (Pleth), ansteigender Hautwiderstand (EDA), gleichmässigere, synchrone, thorakale und abdominale Atembewegung und Einfluss auf die Sympathiko-Vagale-Balance (SVB: errechnet aus Herz- und Atemtätigkeit), die das sympathische und parasympathische Gefüge darstellt. Dies sind Anzeichen für eine tiefere, vegetative Entspannung [27]. Der entspannte Wachzustand wird exemplarisch anhand der Herzfrequenz deutlich. Die Varianzanalyse ergibt für die vegetativen $\mathrm{Pa}$ rameter im Unterschied $\mathrm{zu}$ den vier Messphasen eine signifikante Veränderung ( $\mathrm{p}$-Wert=0,000), beispielsweise $\mathrm{p}$ Wert der Herzfrequenz $(p=0,000)$ im Bezug auf die unterschiedlichen Versuchsphasen.

Die Herzfrequenz in den drei Phasen während $(p=0,000)$, nach der Entspannung $(\mathrm{p}=0,000)$ und während des Wahrnehmungsversuches (WN) $(p=$ 0,038) unterscheidet sich signifikant von der vorher-Phase. Es ist eine deutliche Entwicklung der Herzfrequenz im Verlauf anhand der Abweichung (errechnet durch die Differenz) zu ersehen. In den Phasen während und nach der Entspannung ist die Herzfrequenz kleiner als in der vorher-Phase, in dem Wahrnehmungsversuch (der WN-Phase) liegt die Herzfrequenz dagegen über der Herzfrequenz der vorher-Phase. Bei der Interaktion der Phasen der Entspannung auf die Wahrnehmungsqualität zeigt sich eine Tendenz, die trotz der Streuung eine charakteristische Verteilung der Herzfrequenz bei den Probanden mit unterschiedlicher Wahrnehmungsqualität aufweist. Bei Probanden mit hoher Wahrnehmungsfähigkeit liegt tendenziell $(p=0,151)$ eine niedrigere Herzfrequenz im Entspannungszustand vor, als bei Probanden mit niedriger Wahrnehmungsfähigkeit. (Abb. 2)

Globale kortiko-kortikale Kohärenz Im EEG lässt sich während der erweiterten Wahrnehmung ein neuronales

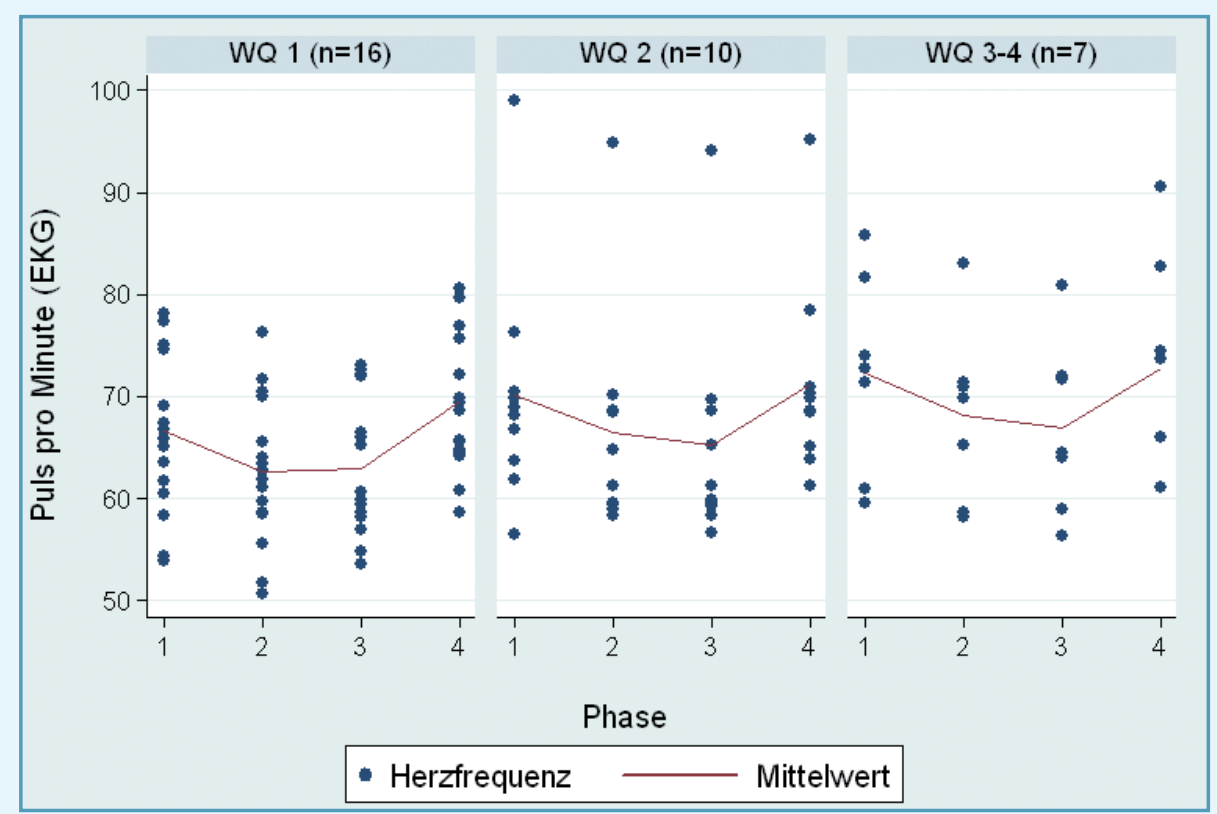

Abb. 2. Verteilung des vegetativen Mittelwertes der Herzfrequenz pro Minute im Verlauf der vier Messphasen vor (1), während (2), nach Ergosoma (3) und während des Wahrnehmungsversuches (4) in Bezug zur Wahrnehmungsfähigkeit der Probanden mit sehr guter (WQ1), guter (WQ2) und niedrigerer (WO3-4) Wahrnehmungsqualität.

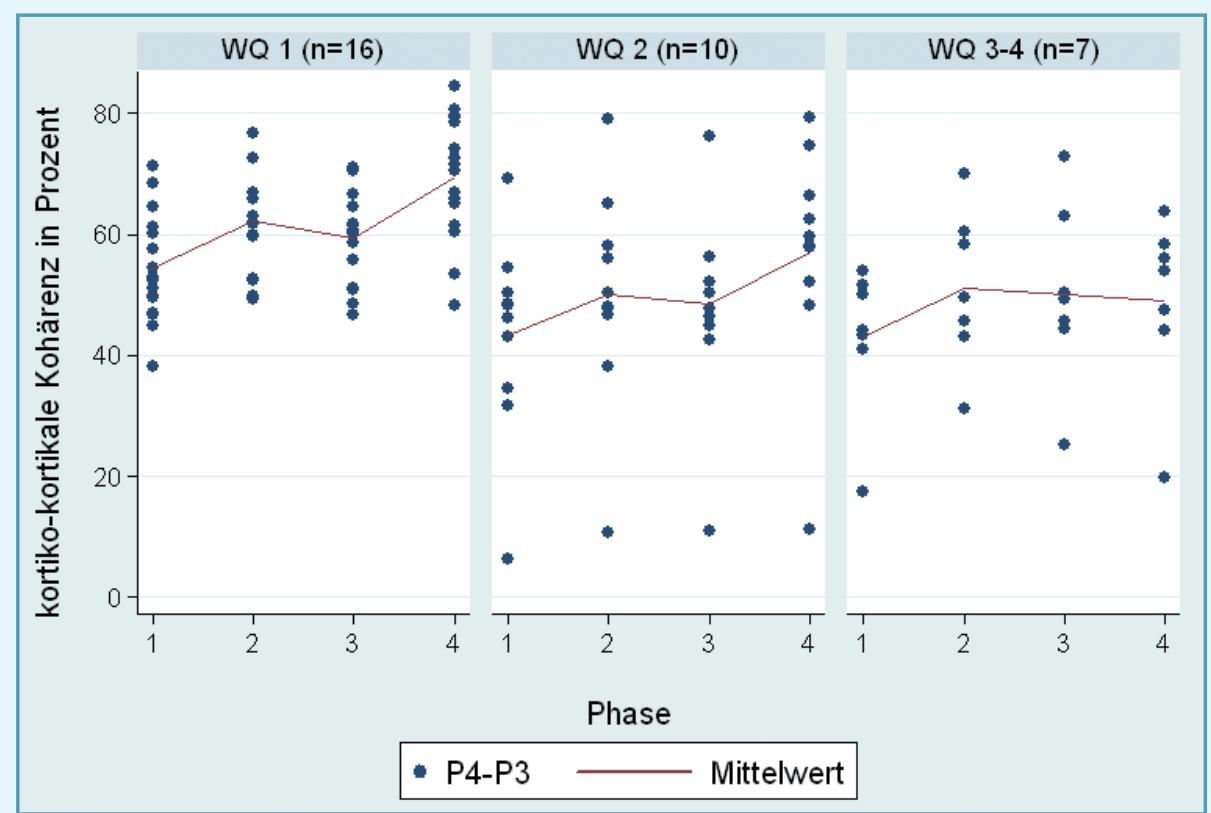

Abb. 3. Verlauf des Mittelwertes der kortiko-kortikalen Kohärenz auf parietaler Ebene in den vier Messphasen vor (1), während (2), nach Ergosoma (3) und während des Wahrnehmungsversuches (4) in Bezug zur Wahrnehmungsfähigkeit der Probanden mit sehr guter (WQ1), guter (WQ2) und niedrigerer (WO3-4) Wahrnehmungsqualität.

Korrelat feststellen, das durch ein spezielles Frequenzspektrum und eine zunehmende Vernetzungsaffinität gekennzeichnet ist [28]. Dieser Zustand erhöhten Bewusstseins zeichnet sich durch eine signifikante Zunahme der kohärenten Momente in der Hirnrinde aus. Gemessen wurde sekündlich ein Kohä- renzwert auf den vier Kortexebenen frontal, zentral, parietal und okzipital, der prozentual ins Verhältnis der Versuchsphasen gesetzt wurde. Somit handelt es sich um eine prozentuale Kohärenz. Die Varianzanalyse zeigt, dass es einen signifikanten Unterschied zwischen den Wahrnehmungsgruppen 


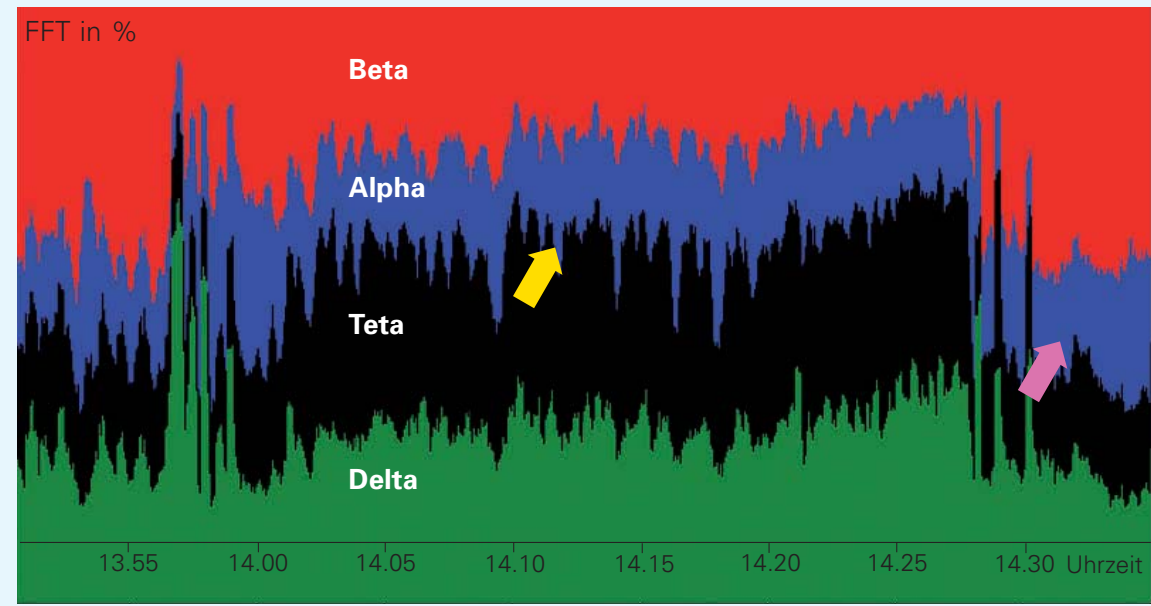

Abb. 4. FFT-Gesamtspektrum der EEG-Rohdaten auf der okzipitalen Ebene über den gesamten Versuch mit den vier Messphasen hinweg. Die FFT-Aufzeichnung zeigt deutlich den steigenden prozentualen Anteil der langsamen Delta-, Theta- und Alphafrequenzen am Gesamtspektrum während der Entspannungsphase (gelber Pfeil) und den zunehmenden AlphaAnteil während des Wahrnehmungsversuches (violetter Pfeil)

WQ1 und WQ4 bei dem zerebralen Parameter der kortiko-kortikalen Kohärenz im EEG gibt ( $\mathrm{p}=$ Wert auf der zentralen Ebene 0,018 und auf der parietalen Ebene 0,020). Kohärenz bedeutet das gleichzeitige, gleichförmige Ausschlagen und Schwingen der Gehirnaktivitäten in verschiedenen Gehirnbereichen oder die Vernetzungseigenschaft der Hirnregionen, hier bezogen auf die Hirnaktivität im Kortex. Die intensivierte Kommunikationsfähigkeit der Hirnareale ist im EEG durch erhöhte Vernetzungseigenschaften und eine höhere Flexibilität der Interaktion von Hirnregionen messbar [29]. Es zeigt sich in den zerebralen Messergebnissen während der Entspannung und vertiefter sensomotorischer und visueller Wahrnehmungseindrücke eine kortiko-kortikale Zunahme der kohärenten Momente bis zu 85\%. (Abb. 3)

Die signifikante Zunahme der kohärenten Momente in der Gehirnrinde entspricht der zurzeit geltenden Erkenntnis und Lehrmeinung der Neurowissenschaft als Anzeichen für bewusstseinserweiternde Zustände und sind nach dem Hirn- und Neurophilosophen Metzinger Bedingungen für die Erweiterung der Wahrnehmungsspektren und der geistigen Leistungen. Die geistige Leistungssteigerung der subtilen Wahrnehmungsfähigkeit wird auf das empathische Wahrnehmen übertragen [30].

\section{Abnehmender Einfluss des Bewertungssystems}

Die gemessene signifikante, global zunehmende Kohärenz im Kortex geht bei einem Vergleich der Wahrnehmungsgruppen WQ1-2 und WQ3-4 $(\mathrm{p}=0,006)$ mit einer Verlagerung der Reizverarbeitung bzw. Hirnaktivität einher. Der Moment einer zunehmenden Neutralität und einer erhöhten Offenheit zeigt sich durch die vermehrte Verlagerung der Aktivität in der Kortexregion (statt in die subkortikalen Areale), was einem verminderten Einfluss des Bewertungssystems entspricht. Der wahrnehmende Arzt ist in diesem Moment von festen Vorstellungen und voreingenommenen Wertungen unabhängiger. Dies wird als neutrale Haltung gegenüber dem Patienten gedeutet. Vorgefasste Meinungen, Sympathien und Antipathien spielen in diesem Fall weniger eine Rolle. Dies ist während erweiterter Wahrnehmung gewährleistet, da dominante Muster in den Hintergrund treten, statt starre Denk- und Empfindungsfähigkeit. Es entsteht während des empathiefähigen Handelns eine Integrität, die nötig ist, damit eine erweiterte Wahrnehmung einsetzen kann und Empathie funktioniert. Dies gilt für Patienten wie Behandler [31].

\section{Frequenzspektrum}

Durch ein Frequenzspektrum aus hohen Anteilen an Delta-, Theta- und Al- phawellen in Kombination mit Betawellen kann die geistige Entspannung im EEG (Elektroenzephalografie) festgestellt werden. Eine entspannte, mentale Grundhaltung ist die Voraussetzung für eine umfassende Wahrnehmung, wie sich an der spezifischen $\mathrm{Zu}$ sammensetzung des Wellenspektrums zeigt. Durch die Frequenzanalyse des EEG (FFT) kann die Zusammensetzung der Frequenzanteile im EEG ermittelt werden. Die Fourier-Transformation (FFT) ist eine effiziente Berechnung zur Frequenzanalyse des EEG und damit der vorhandenen Wellenspektren $\mathrm{zu}$ einem bestimmten Zeitraum im EEG, errechnet durch einen Algorithmus, um die Verteilung der Wellenspektren in ihren Anteilen von 100\% angeben zu können. Während der erweiterten Wahrnehmung fällt die auffällige Kombination von schnellen Betawellen auf, die bei Wachheit in Kombination mit hohen Anteilen an langsameren Alpha- und Thetawellen für die Empfindungsfähigkeit auftreten. Eine derartige Verteilung der Wellenspektren ist für ein Bewusstsein konzentrierter und zugleich entspannter, offener Aufmerksamkeit charakteristisch. In der Forschungsarbeit wird diese höhere Achtsamkeit der Wahrnehmung als kritische Beobachtungsgabe bezeichnet. Diese bewusste Aufmerksamkeit ist Voraussetzung für eine mental entspannte, empathiefähige Grundhaltung, die eine gesamtheitliche Wahrnehmung des Patienten und seiner Umgebung zulässt und somit Teil der ganzheitlichen Medizin ist. Bei konzentrierter, angestrengter, fokussierter Betrachtung dagegen tritt ein dominantes Betaspektrum auf und die Anteile der Thetaund Alpha-Wellen nehmen stark ab.

Bei einem somnolenten Zustand würde das Delta-Spektrum zunehmen und der Anteil der Theta-Alphaspektren ebenfalls geringer ausfallen. Das Besondere der vertieften Betrachtungsform mit hoher Sensibilität ist, dass trotz offener Augen die Alpha-Blockade [32] aufgehoben wird, was ein Anzeichen für Entspannung im Wachzustand ist (Abb. 4). Dagegen tritt bei konzentrierter, fokussierter Betrachtung ein dominantes Betaspektrum auf und die Anteile der Alpha-Wellen nehmen stark ab. Die Messergebnisse deu- 
ten auf eine kontemplative, wache Entspannungsphase mit einer hohen Aufmerksamkeit und gleichzeitiger innerer, körperlicher und mentaler Ruhe hin. Der Arzt ist in diesem Zustand ein kritischer Beobachter mit grosser Offenheit und Neutralität, entspannt und hellwach.

\section{Vegetative Stabilität und zerebrale Flexibilität}

Eine hohe Wahrnehmungsleistung geht mit einem besonderen, ausgewogenen vegetativen Entspannungszustand einher. Es ist anhand der Abweichung oder Differenz der Mittelwerte ein Trend bei den Parametern der Herzfrequenz (Hf), der Herzfrequenz-Variabilität (HRV) und der Sympathikus-Vagus-Balance (SVB) sichtbar, dass sich die Probanden mit hoher Wahrnehmungsqualität ausgewogener und stabiler entspannt haben. Den Unterschied der einzelnen Werte zum Mittelwert nennt man Abweichung vom Mittelwert. Die Probanden mit hoher Wahrnehmungsqualität zeigen im Vegetativum eine niedrigere Abweichung (Differenz der Mittelwerte) auf, als Probanden mit niedriger Wahrnehmungsqualität. Die Messergebnisse zeigen, dass sich das vegetative Nervensystem hier in einem dynamischen Balancezustand befindet, was weiter die Wachheit für die kognitiven Leistungen aufrecht erhält. Die Probanden mit einer niedrigen Wahrnehmungsqualität besitzen dagegen grössere Abweichung (Differenz der Mittelwerte) von der Phase Entspannung zur Phase des Wahrnehmungsversuches, wie beispielsweise beim Spektrum der Sympathikus-Vagus-Balance (Abb. 5) oder der Herzfrequenz.

Auf zerebraler Ebene sieht es anders aus, denn hier kennzeichnet eine grosse Abweichung (Differenz der Mittelwerte) den Zustand der hohen Wahrnehmungsqualität. Dies zeigt, wie starr und unausgewogen oder flexibel und ausbalanciert die vegetativen und zerebralen Funktionszustände sind. Die Abweichung (Differenz der Mittelwerte) lässt sich als zunehmende Flexibilität der geistigen Leistungen und zunehmende kohärente Vernetzungseigenschaften deuten (Abb. 6).

Die Messergebnisse zeigen eine kon-

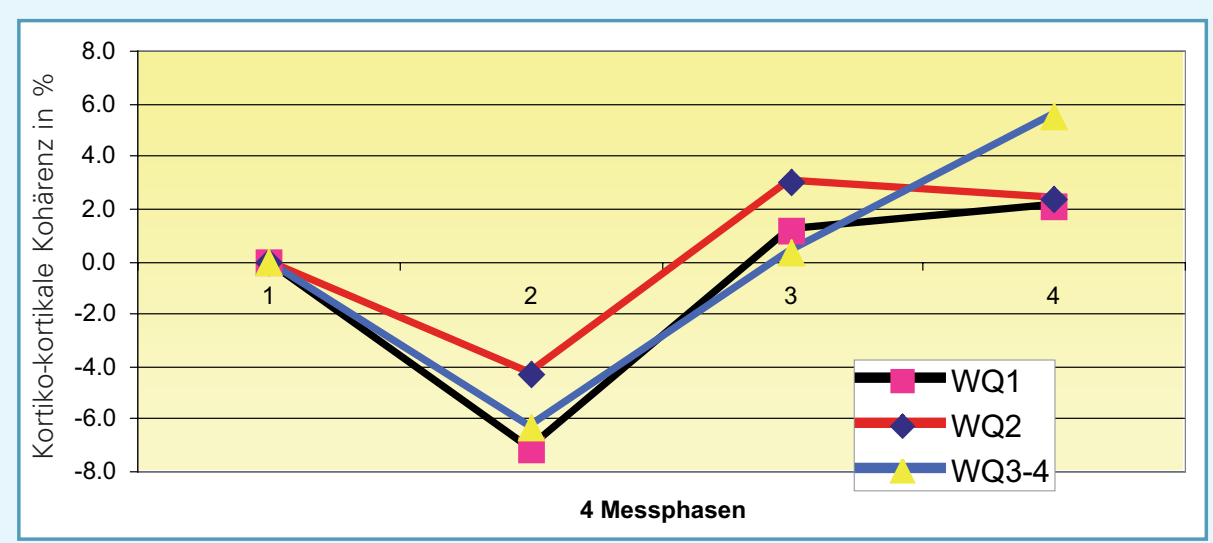

Abb. 5. Das Spektrum der Sympathikus-Vagus-Balance (errechnet aus der Korrelation von Herzund Atemaktivität und den Abweichungen aus den Minimal- und Maximalwerten) im Verlauf der vier Messphasen vor (1), während (2), nach Ergosoma (3) und während des Wahrnehmungsversuches (4) in Bezug zur Wahrnehmungsfähigkeit der Probanden mit sehr guter (WQ1), guter (WO2) und niedrigerer (WO3-4) Wahrnehmungsqualität.

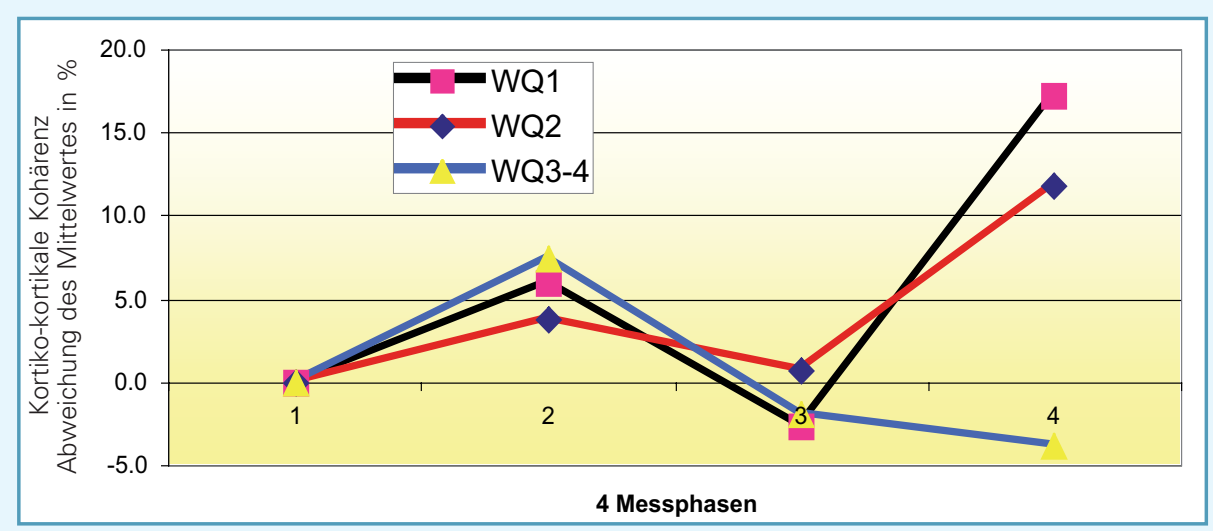

Abb. 6. Das Spektrum der globalen, kortiko-kortikalen Kohärenz (errechnet aus den Abweichungen (Differenz) der Minimal- und Maximalwerte) im Verlauf der vier Messphasen vor (1), während (2), nach Ergosoma (3) und während des Wahrnehmungsversuches (4) in Bezug zur Wahrnehmungsfähigkeit der Probanden mit sehr guter (WQ1), guter (WQ2) und niedrigerer (WO3-4) Wahrnehmungsqualität.

templative, wache Entspannungsphase mit einer hohen empathiefähigen Aufmerksamkeit und gleichzeitiger innerer, körperlicher und mentaler Ruhe auf. Das Vegetative und Zerebrale steht in einer engen homogenen Verknüpfung, wenn ein subtiler Wahrnehmungseindruck entsteht. Das bedeutet, dass der Arzt aus einer körperlichen Stabilität und einer mentalen Offenheit heraus vertieft und bewusst, subjektiv wahrnimmt, was einen prägenden Einfluss auf Diagnose, Behandlung und die Arzt-Patienten-Beziehung haben kann.

\section{Kreativität messbar durch Abweichung der Mittelwerte}

Kreativität ist als geistige Funktion durch eine hohe Flexibilität des Geistes ausgezeichnet. Während des vertieften Wahrnehmungserlebnisses zeichnet sich das Gehirn durch eine hohe Flexibilität der Vernetzung von Neuronenkoalitionen aus. Diese Deutung lässt sich aus der kortiko-kortikalen Kohärenz ableiten $(p=0,006)$ [33]. Die Denkstruktur als geistige Flexibilität folgt während erweiterter Wahrnehmungsspektren nicht mehr ausschliesslich alten, eingefahrenen Bahnen, sondern formt sich neu, was man auch als Hirnplastizität bezeichnet. Es wird angenommen, dass sich die Hirnaktivität bzw. die Reizverarbeitung in diesem Zustand verstärkt verändern und anpassen kann. Dabei entstehen Erregungsmuster im neuronalen Netzwerk des Gehirns, die nicht mehr auf geordneten Bahnen basieren. Im Moment des offenen Wahrnehmens verändern und modifizieren sich die neuen Nervenaktionspotentiale, die veränderte 


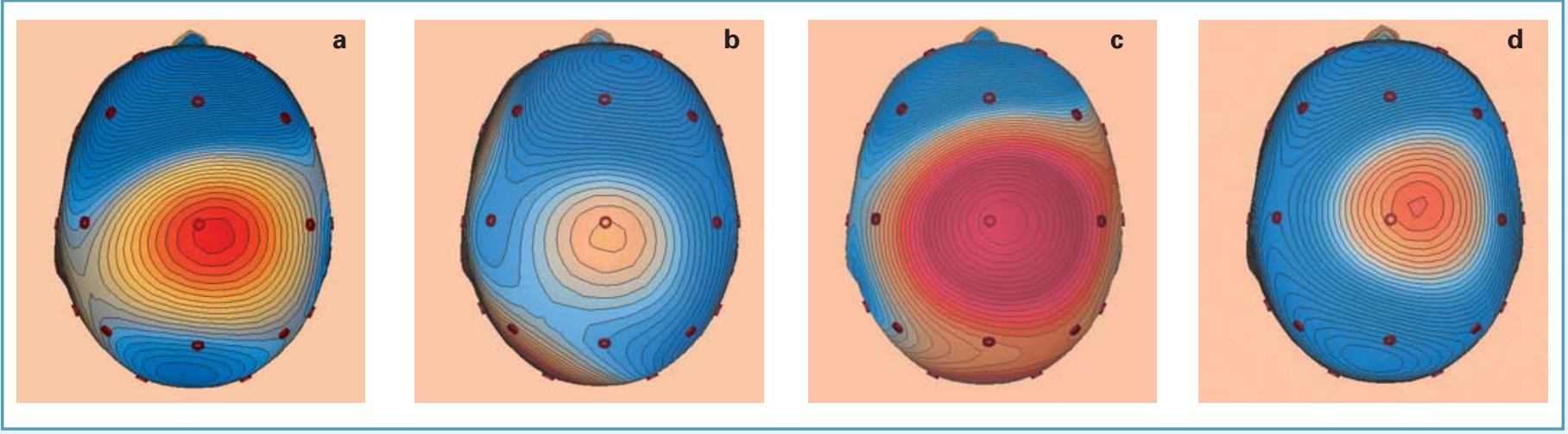

Abb. 7. Brain-Mapping einer konzentrierten Hirnaktivität im On-Off-Effekt als sich ständig modifizierendes, wechselndes und wiederkehrendes Aktivierungsniveau im parietalen Bereich des Kortex über mehrere Sekunden. Es wird die Verteilung der Hirnstrom-Aktivität des Kortex dargestellt (rot = maximale Aktivität, blau = minimale Aktivität). Hohe Hirnaktivität ist an einer intensiv roten Farbe im Brain Mapping ersichtlich. 7a zeigt den On-Effekt, 7b den Off-Effekt 160ms später, 7c zeigt den On-Effekt wieder 200ms später und 7d den Off-Effekt 100ms später. Das Brain-Mapping zeigt eine parietale Aktivierung im Kortex eines Probanden mit hoher Wahrnehmungsfähigkeit während des Wahrnehmungsversuches. Die Konzentration der Aktivität im Vertex steht für einen intensiven Wahrnehmungseindruck, der sich konstant durch den kontinuierlichen On-Off-Effekt hält. In diesem Areal des Parietallappen liegt das funktionelle Verarbeitungszentrum für die somatosensorische Empfindung und Integrationsleistung von visuellen und somatosensorischen Reizen.

Neuronenkoalitionen und Verknüpfungen eingehen. Eine temporale und frontale Aktivierung, als Aufmerksamkeitssteigerung gedeutet, kann sich von einem lateralen Aktivierungszustand zu einem kohärenten Zustand und einer Integration zentraler und parietaler Areale während vertiefter, empathiefähiger Wahrnehmung verändern. Im Zustand der Kreativität scheint das Gehirn neue Denkpfade einzuschlagen. Kreativität ist ein Anzeichen dafür, dass sich das Denken verändert und vor allem frei und offen geworden ist.

\section{Wahrnehmung als Kennzeichen der Empathiefähigkeit}

Die Probanden haben im Anschluss an die Entspannungsphase über intensive, sensomotorische, körperliche und mentale emotionale Empfindungen berichtet. Die Anzahl der wahrgenommenen Phänomene wie die visuellen Modifikationen von drei Kreisen, einem dreidimensionalen Eindruck des mittleren Kreises der Tafeln von Chartres, der auch zunehmend mit einer Corona als Umriss zusammenfallen kann, sowie sensomotorischem Körperempfinden wie Pulsieren oder ein Wärmeeffekt in Gewebestrukturen nehmen im Wahrnehmungsversuch zu, wenn ein entspannter Wachzustand vorliegt.

\section{Parietale Konzentration der Hirnaktivität im Brain-Mapping}

Es kann während einer umfassenderen Betrachtungsweise eine konzentrier- tere Hirnaktivität im parietalen Bereich des Kortex (zum Scheitel gehörig), also im Vertex, durch das BrainMapping festgestellt werden. Die Aktivierung dieses Bereiches steht für die Verarbeitung körperlichen, sensomotorischen Empfindens, der Fähigkeit zur Integration von Reizen und der Integrationsleistung von Informationen $\mathrm{zu}$ einem Gesamteindruck [34]. Die parietale Ebene ist ein Ort für die Verknüpfung unterschiedlicher Sinneseindrücke, der sogenannten Synästhesien [35]. Dieses integrative Denken durch konzentrierte, globale, kohärente Aktivität im parietalen Bereich des Kortex als geistige Leistung entwickelt sich aus der mentalen Ausrichtung der Aufmerksamkeit für einen erweiterten Wahrnehmungseindruck [36]. Gleichzeitig wird dieser Moment als ein Einheitsgefühl erlebt, bei dem der Proband den Eindruck einer komplexen Wahrnehmung schildert, was mit einem losgelösten Zeitgefühl und einem Erleben des Augenblicks verbunden ist.

\section{Kontinuität der Wahrnehmung durch den On-Off-Mechanismus}

Das integrative Denken und Empfinden eines unmittelbaren Momentes mit einem veränderten Zeitempfinden ist auf physiologischer Ebene durch die Messungen der Forschungsreihe nachvollziehbar. Auf der zerebralen Ebene ist bei intensiven Wahrnehmungserlebnissen von Probanden messtechnisch ein On-Off-Mechanismus im Brain-Map- ping zu erkennen. Bei einer zunehmenden Wahrnehmungsqualität (WQ) steigt die immer wiederkehrende Aktivierung im On-Off-Effekt besonders parietal an, nimmt wieder ab und an demselben Ort wieder zu. Dies zeigt eine Konstanz im Wandel, zur gleichen Zeit eines intensiven Wahrnehmungserlebnisses. Die Messungen des Brain-Mappings fanden im Millisekundenbereich statt und nach Beobachtung mit dem Auge wurden die Veränderungen der Hirnaktivität mit deren Intensität und lokalem Auftreten festgestellt. Dazu wurden die Messungen im Millisekundenbereich durchgesehen und beispielhafte Abbildungen der Aktivitätsverläufe ausgewählt (Abb. 7). Während der Anwendung der Ergosoma-Methode und dem anschliessenden Wahrnehmungsversuch konnte ein Moment gemessen werden, bei dem das zeitliche und räumliche Wahrnehmungselement verändert wird. Der offene Zustand zeigt ein neues Zeitempfinden auf. Es konnte während vertiefter Wahrnehmung mit der entspannenden Ergosoma-Anwendung und dem Schauen im Wahrnehmungsversuch eine veränderte Informationsverarbeitung nachgewiesen werden. Während die Probanden von einem zeitfreien Erleben sprechen, hebt sich der typische 3-Sekunden Rhythmus der alltäglichen Informationsverarbeitung auf [37]. Alle drei Sekunden sucht üblicherweise, evolutionär geprägt, unser Sinnessystem nach neuen Informationen bzw. 
Reizen. Dieser Takt der Sinne hebt sich im Moment der erweiterten Wahrnehmung zugunsten eines kontinuierlich andauernden Momentes (bis 12 Sekunden andauernd gemessen) durch den On-Off-Mechanismus der Gehirnaktivität auf. D.h. ein andauerndes An und Aus der Hirnaktivitäten an einem konstanten Ort des Kortex hält über mehrere Sekunden als Zeichen eines konstanten Erlebens an. Während empathisch geprägtem Handeln entsteht ein momentanes, gegenwärtiges, einfühlendes Gewahrsein.

Der vertiefte Eindruck der Wahrnehmung kann auf diese Weise über mehrere Sekunden hinweg durch $\mathrm{Zu}$ und Abnehmen des Aktivitätsniveaus in einem konzentrierten Hirnbereich des Kortex kontinuierlich gehalten werden. Dies entspricht einem tiefen Wahrnehmungserlebnis, das als Gegenwärtigkeit des Behandlers während der unmittelbaren Betrachtung des Patienten gedeutet werden kann [38]. Dieser zerebrale Zustand stellt eine günstige Bedingung dar, bei der die kreative Arbeit nicht zu Ermüdung führt. Arzt und Therapeut gewinnen aus der Verrichtung ihrer darüberhinaus qualitativer ausgeführten Aufgabe Energie.

\section{Diskussion}

Die Forschungsarbeit zeigt, welche Bedingungen für Empathie nötig sind, damit die Ergebnisse zur erweiterten Wahrnehmung auf die Empathie eines Arztes oder der Arzt-Patienten-Beziehung übertragen werden können. Es wird aufgezeigt, dass vegetative Entspannung und kortiko-kortikale globale Kohärenz (Vernetzungseigenschaft im Kortex) die Wahrnehmungsfähigkeit der Probanden fördert und bewusstseinserweiternd wirkt. Dies kann bedeuten, dass bewusstseinserweiternde Zustände eine Empathiefähigkeit fördern. In einem entspannten Wachzustand ist die Wahrnehmung verbessert, was die Qualität der Behandlung steigert. Es sollte also auf den eigenen emotionalen und mentalen Zustand geachtet werden. Die psycho-physische Entspannungsfähigkeit gehört in diesem Sinne zu den Kompetenzen, sie kann z.B. mit der Ergosom-Methode geschult werden. Methoden zur Wahrnehmungsschulung sind u.a. Körperübungen mit Atemlenkung und Aufmerksamkeitsschulung (z.B. bei Qi Gong, Yoga, Feldenkrais und Ähnlichem).

Eine erweiterte Wahrnehmung hat nachweislich einen Einfluss auf die Empathiefähigkeit. Das Empathievermögen und die eigenen Ressourcen entwickeln sich durch einen entspannten vegetativen und geistigen Zustand. Diese Haltung versetzt in die Lage, die Situation des Patienten umfassender und tiefer zu erkennen, was im Wahrnehmungsversuch mit der gelungenen Betrachtung, also dem Erkennen der Phänomene eines Doppelbildes, vergleichbar ist. Der Wahrnehmungsversuch mit der Betrachtung der gegensätzlichen Farben und Formen der Tafeln von Chartres, die bei hoher Wahrnehmungsqualität zu einem neuen, phänomenalen Farbeindruck fusioniert werden können, kann als Indiz für die Tiefe des Empfindens und Wahrnehmens auf die Arzt-Patienten-Situation übertragen werden.

Diese Phänomene treten im Wahrnehmungsversuch auf, wenn der binokulare Wettbewerb bei Darstellung von zwei verschiedenfarbigen Kreisen im Gehirn verarbeitet und durch den Eindruck eines dritten Kreises in einer Mischfarbe gelöst wird. Der Blick ist entspannt auf das Zentrum gerichtet, in der Diagnose oder Behandlung übertragen beispielsweise auf die Erfüllung einer Aufgabe wie die Blutdruckmessung und eine gleichzeitigen Erfassung der Gesamtsituation und Befindlichkeit des Patienten. Der mentale Zustand einer offenen Aufmerksamkeit versetzt den Behandler in die Lage, Details und die Gesamtsituation bewusst zu registrieren. Somit kann durch die gewonnene Neutralität gelassen und individuell auf den Patienten reagiert werden. Es entsteht eine umsorgende Nähe mit einer gesunden Distanz durch die vegetative und zerebrale Entspannung des Arztes und Therapeuten. Empathie darf jedoch nicht verwechselt werden mit Mitgefühl oder Sympathie.

Eine derartige Verarbeitung ist vergleichbar mit einem von Empathie geprägten Denken und Handeln, wenn der Gesamtzusammenhang komplexer
Inhalte erschlossen wird und Details sowie die Situation und das Umfeld des Patienten als Ganzes erkannt zu einer Lösung geführt werden. Hebt sich das dual-polare Denken über das Entweder-Oder von Gesundheit und Krankheit hinaus, tritt an diese Stelle ein integratives Denken und ein kreatives Ahnen des gegenwärtigen Moments, in dem Gesundheit nicht willentlich produziert wird, sondern entstehen kann.

Das Wahrnehmungsspektrum braucht der Arzt zur Gestaltung der Arzt- und Patienten-Beziehung. Wenn er sich einfühlt, spürt der Patient dies umgehend und gewinnt Vertrauen. Durch eine bewusst gestaltete Beziehung kann der Arzt ein vertrauensvolles Klima schaffen, in dem Ressourcen geweckt werden, die den Patienten in der Auseinandersetzung mit der Situation von Erkrankung oder des Gesundwerdens stärken. Es entsteht Vertrauen und eine wechselseitige sich einlassende Beziehungsfähigkeit. Der Arzt nutzt die Subjektivität, um ein Gesamtbild zu erhalten. Eine erweiterte Wahrnehmung unterstützt das Einbeziehen eines kreativen Teils in der Diagnose und Behandlung.

Wenn die Wahrnehmung von Arzt und Therapeut entspannt und wach zugleich ist, findet leichter eine Ergänzung zum Ansatz der Pathogenese durch die Salutogenese statt. Es ist eine Aufmerksamkeit, mit der phänomenologisch und annähernd wertfrei die Dinge betrachtet werden, wodurch das Phänomen von Gesundheit wahrscheinlich deutlicher erfahren werden kann. Die Ergebnisse zeigen, dass Behandlung und Wahrnehmung Zeit brauchen. Zum einen Zeit für den Patienten und zum anderen Zeit für die mentale Ausrichtung des Arztes und Therapeuten. Der Arzt und Therapeut als Wahrnehmender nutzt seine bewusste, mentale Ausrichtung, um den Patienten zu erkennen. Er entwickelt Intuition und Feinsinn, um den kreativen Prozess der Heilung zu erkennen und dadurch zu fördern. Diese Fähigkeit wird durch zunehmende Anzahl der Momente einer kortiko-kortikalen Kohärenz beim Wahrnehmenden gefördert.

Die vorliegende Forschungsarbeit stellt die Bedeutung der umfassenden 
Wahrnehmung des Arztes und Therapeuten für eine effektive Tätigkeit in der Interaktion zum Patienten heraus und weist somit auf die Bedeutung eines achtsamen Umgangs mit der körperlichen und mentalen Verfassung beim Arzt und Therapeuten hin. Die eigenen Sinne werden klarer wahrgenommen, die eigene Situation wird eher reflektiert und die Bereitschaft zur eigenen Selbstpflege wird gefördert [39]. „Denn das, was ich für mich wahrnehme, kann für den anderen hilfreich sein.“ Persönliches Achtsamkeitstraining ist ein wichtiges Hilfsmittel zur eigenen Stressbewältigung, Prävention und Gesundheitspflege.

\section{Wohlbefinden des Arztes durch empathisches Handeln}

Die Herausforderung im ärztlich-therapeutischen Alltag bleibt die Offenheit, die neben Fachwissen und Standardisierungen ein Wahrnehmen des Patienten integrieren, bei der der Patient in einer grösseren Weite wahrgenommen werden kann. Obwohl der Arzt Sachzwängen unterliegt, die ihm vielleicht inneren Druck bereiten, bietet ihm Empathie etwas, wodurch er neue Inspiration im Arbeitsalltag erlebt. Es geht dabei weniger um das ärztliche Berufsfeld mit dem jeweiligen Handwerkszeug, als um die Subjektivität des Arztes als zusätzlich nutzbares Werkzeug und die Qualität der Weite als inneres Empfinden und Lebensgefühl, die er in seine Arbeit mit einbezieht. Es steht also das Wohlbefinden des Arztes und Therapeuten im Blickpunkt der Aufmerksamkeit und Wertschätzung. Empathie stellt eine Bereicherung und eine Entlastung für das Arbeitsgefühl des Arztes zur Verfügung. Das Distanz-Nähe-Prinzip ist therapeutisch und professionell, aber die Empathie ruft eine Lebendigkeit für den Arzt hervor, die eine Unterstützung für seinen Alltag darstellt. Der Arzt steht auch immer wieder vor einem Abenteuer des Lebens und dem Wunder des Lebens gegenüber, für das er sich auf seine Methode einsetzt. Er bewahrt sich mit dem Bewusstsein für eine erweiterte Wahrnehmung die Fähigkeit des Staunens und Erstaunens.

\section{Literatur}

1. von Weizsäcker V: Der Gestaltkreis: Theorie der Einheit von Wahrnehmung und Bewegung. Thieme Verlag, Stuttgart 1947.

2. Hensel H: Allgemeine Sinnesphysiologie. Hautsinn, Geschmack, Geruch. Springer Verlag, Heidelberg, Berlin 1966.

3. Becker K, Sachse R: Therapeutisches Verstehen. Hogrefe, Göttingen 1998.

4. Schönberger, Alwin: Patient Arzt. Der kranke Stand. Wien 1995.

5. Otte R: (2001). Thure von Uexküll. Von der Psychosomatik zur Integrierten Medizin. Vandenhoeck \& Ruprecht, Göttingen 2001

6. Speierer G-W: Das patientenorientierte Gespräch. Baustein einer personenzentrierten Medizin. Causa, München 1985

7. Dürholtz D. Zur Kommunikation zwischen Arzt und Patient. Peter Lang, Frankfurt/M 1993.

8. Matthiessen P F: Pluralität - auf dem Weg zu einer Integrativen Medizin? Forschende Komplementärmedizin/Research in Conplementary Medicine 2002;15(5):248-250.

9. Bocia M, Campos J J: Mental emotional signals, social referencing, and infants reactions to strangers. In: Eisenberg N (Hrsg.), Empathy and related emotionals responses. JosseyBass, San Francisco 1989, S. 25-49

10. Antonovsky A: Salutogenese, Zur Entmystifizierung der Gesundheit. DGVT-Verlag, Tübingen 1997.

11. Brieskorn-Zinke M: Die pflegende Relevanz der Grundgedanken des Salutogenese-Konzepts. Pflege. Die wissenschaftliche Zeitschrift für Pflegeberufe 2000;13:373-380

12. Matthiessen P F: Perspektivität und Paradigmenpluralismus in der Medizin. In: Fuchs B, Kobler-Fumasoli N (Hrsg.): Hilft der Glaube? LIT-Verlag, Münster 2002, S. 3-35

13. Brothers L: A Biological Perspective on Empathy. Am. J. Psychiatry 1989;1:10-19.

14. Gilch-Geberzahn G: Vom Wahrnehmen zum Deuten. Über die innere Arbeit des Therapeuten. Forum Psychoanalyse, 1998;14:34-51.

15. Kohut $\mathrm{H}$ : Introspection, empathy, and the semicircle of mental health. In: Lichtenberg J, Bornstein M, Silver D (Hrsg.): Empathy I. Analytic Press, Hillsdale, NJ 1984 (S. 81-100)

16. Bergner Th: Burn-out bei Ärzten: Lebensaufgabe statt Lebens-Aufgabe. Dtsch Arztebl 2004;101(33):A-2232/B-1866/C-1797.

17. Gadamer H-G: Über die Verborgenheit der Gesundheit. Suhrkamp, Frankfurt a.M.1993.

18. Dörner K: Der gute Arzt: Lehrbuch der ärztlichen Grundhaltung. Schattauer Verlag, Stuttgart 2003.

19. Gebser J: Ursprung und Gegenwart des integralen Bewusstseins. Novalis, Schaffhausen 1986.

20. Baumeister W: Das Unbekannte in der Kunst. Schwab, Stuttgart 1947.

21. Pennington G: Die Tafeln von Chartres. Walter-Verlag, Düsseldorf 1994.

22. Ditzinger Th: Illusion des Sehens. Elsevier, München 2006.

23. VanRullen R, Reddy $L$, Koch C: Visual search and dual-task reveal two distinct attentional resources. Journal of Cognitive Neuroscience 2004;16(1):4-14

24. Kaplan S: Neurophysiologische Korrelate malerischer Begabung - Eine kognitionsneurowissenschaftliche Studie mittels EEG. Wien 1998, Universität, Institut für Neurophysiologie / Kaplan S: Visuelle Wahrnehmung und bildliches Vorstellen, zwei Aspekte einer komplexen Begabung - eine EEG-Amplituden- und Kohärenz-Studie. Wien 1995.

25. Petsche H, Kaplan S, von Stein A, Filz O: The possible meaning of the upper and lower alpha frequency bands for cognitive and creative tasks. Edited by Basar E, Hari R, Lopes da Silva FH, Schürmann M, In: Brain alpha activity - New aspects and Functional Correlates. Int Journal of Psychophysiology 1997;26:77-97.

26. Metzinger Th: Ganzheit, Homogenität und Zeitkodierung. In: Bewusstsein - Beiträge aus der Gegenwartsphilosophie. mentis, Paderborn 1995

27. Schandry R: Biologische Psychologie, in: Perspektiven der Psychologie. Eine Standortbestimmung. Weinheim 1996, S. 65-83 und Boucsein 1988

28. Bhattacharya J, Petsche $\mathrm{H}$ : Shadows of artistry: cortical synchrony during perception and imagery of visual art. Brain Research Cognitive Research (Netherlands) 2002;13(2).

29. Petsche $H$, Lindner $K$, Rappelsberger $P$, Schmidt-Henrich E: Are there hints of creative power in the EEG? Wien 1988 / von Stein A, Rappelsberger P, Filz O, Petsche H: EEGKorrelate bildlicher Vorstellung: Eine Amplituden- und Kohärenzuntersuchung. Zeitschrift für EEG-EMG 1993;24:217-224.

30. Singer T: The neuronal basis and ontogeny of empathy and mind reading: Review of literature and implications for future research. Neuroscience and Biobehavioral Reviews 2006;30:855-863.

31. Norton WW, Decety J: A social cognitive neuroscience model of human empathy. In: E. Harmon-Jones E \& Winkielman P (Hrsg.): Social neuroscience: Integrating biological and psychological explanations of social behavior. Guilford Press, New York 2007, S. 246-270.

32. Neundörfger B: EEG-Fibel. Das EEG in der ärztlichen Praxis. Urban und Fischer Verlag, München 2002.

33. Singer $W$, Ricard $M$ : Hirnforschung und Meditation. Suhrkamp, Frankfurt am Main 2008.

34. Assad J A Maunsell JHR: Neuronal correlates of inferred motion in primates posterior parietal cortex. Nature 1995;373:518-521 / Fogassi L, Ferrari PF, Gesierich B, Rozzi S, Chersi F, Rizzolatti G: Parietal lobe: From action organization to intention understanding. Science 2005;308:662-667.

35. Aeppli W: Sinnesorganismus, Sinnesverlust. Freies Geistesleben, Stuttgart 1995

36. Koch Ch: Bewusstsein. Ein neurobiologisches Rätsel. Elsevier/Spektrum, München 2005:

37. Pöppel E: Wie kam die Zeit ins Hirn? Neurophysiologische und psychologische Untersuchungen zum menschlichen Zeiterleben. In: Weis K (Hrsg.): Was ist Zeit? Hanser Verlag, München 1995 und Pöppel E: Zum Entscheiden geboren. Hanser Verlag, München 2008.

38. Bieri P: Analytische Philosophie des Geistes. Belitz, Königstein 1981.

39. Milz H: Der wiederentdeckte Körper. Vom schöpferischen Umgang mit sich selbst. Artemis \& Winkler Verlag, München 1992.

\section{Korrespondenzadresse}

Markus Köhl, wissenschaftlicher Mitarbeiter Med. Fakultät der Univ. Witten-Herdecke ZEPU-GmbH, Zentrum für Elektropathologie und Umweltmedizin

Stockumer Str. 28, DE-58453 Witten

mkoehl@web.de 